\title{
Management of Severe Dabigatran-Related Hemorrhage: Case Report, Considerations for Management, and Recommendations
}

\author{
Ian Plener, Sharon Sadry, Lee Mozessohn, Justin Y Lee, Damen Man, Nadine Shehata, \\ and Jeffrey $M$ Singh
}

\section{INTRODUCTION}

$\mathrm{D}^{\prime}$ abigatran is a direct thrombin inhibitor recently approved in the United States and Canada for the prevention of stroke and systemic embolism in nonvalvular atrial fibrillation. Although dabigatran is administered as a fixed dose, does not interact with foods, and has fewer drug interactions than warfarin, the risk of hemorrhagic complications is similar for these two drugs. ${ }^{1,2}$ There is no specific reversal agent for dabigatran, and reversal of coagulopathy in the event of severe hemorrhage can be challenging. With growing use of dabigatran for long-term anticoagulation, it is important that acute care providers have a standardized and evidence-based approach to treating patients with severe hemorrhage secondary to dabigatran. We report a case of severe gastrointestinal hemorrhage related to dabigatran and discuss the management of severe hemorrhage in this context.

\section{CASE REPORT}

An 80-year-old man* presented to a hospital emergency department with a 3-day history of hematemesis, melena, and fatigue. The patient had chronic atrial fibrillation, and dabigatran $150 \mathrm{mg}$ orally twice daily had been prescribed about 16 months earlier. His medical history included osteoarthritis, hypertension, benign prostatic hypertrophy, and hypothyroidism. There was no previous history of alcohol use, liver disease, renal disease, or gastrointestinal hemorrhage. Concurrent medications were celecoxib, lisinopril, digoxin, hydrochlorothiazide, levothyroxine, alfuzosin, and tolterodine. In the month before this presentation, various antibiotics had been prescribed for a urinary tract infection, specifically trimethoprimsulfamethoxazole, nitrofurantoin, ciprofloxacin, and amoxicillin.

\footnotetext{
*The patient provided consent for publication of this case report.
}

In the emergency department, the patient's systolic blood pressure was $85 \mathrm{~mm} \mathrm{Hg}$, heart rate $135 / \mathrm{min}$, temperature $33.3^{\circ} \mathrm{C}$, respiratory rate $35 / \mathrm{min}$, and oxygen saturation $92 \%$ with $5 \mathrm{~L} / \mathrm{min}$ of oxygen by nasal prongs. His abdomen was not tender, and the results of neurological, cardiac, and respiratory examinations were unremarkable. Initial laboratory investigations were remarkable for the following results: hemoglobin $57 \mathrm{~g} / \mathrm{L}$ (normal range 120-160 g/L), serum creatinine 499 $\mu \mathrm{mol} / \mathrm{L}$ (normal range $50-98 \mu \mathrm{mol} / \mathrm{L}$ ), potassium $5.2 \mathrm{mmol} / \mathrm{L}$ (normal range $3.5-5.2 \mathrm{mmol} / \mathrm{L}$ ), international normalized ratio (INR) 7.7 (normal range $0.8-1.2$ ), and activated partial thromboplastin time (aPTT) $122 \mathrm{~s}$ (normal range 26-32 s). The patient was resuscitated with a total of $4.5 \mathrm{~L}$ IV saline and 7 units of packed red blood cells. He received vitamin K $10 \mathrm{mg}$ IV, 5 units of frozen plasma, and $25 \mathrm{IU} / \mathrm{kg}$ prothrombin complex concentrate (Octaplex, Octapharma). Pantoprazole and octreotide were also administered for the gastrointestinal hemorrhage. Hematemesis continued despite these interventions, and, after $8 \mathrm{~h}$ of resuscitation, the anemia and coagulopathy persisted (INR 6).

The patient was transferred directly to the intensive care unit (ICU) of an academic referral centre with ongoing gastrointestinal hemorrhage and hypotension. At the time of the transfer, $12 \mathrm{~h}$ after initial presentation, INR was 4.67, aPTT $114 \mathrm{~s}$, and thrombin time more than $150 \mathrm{~s}$. The patient was given an additional 6 units of packed red blood cells and 4 units of frozen plasma. Emergency endoscopy revealed that the stomach was filled with blood, there was diffuse bleeding from almost all mucosal surfaces, and there was no focal source of hemorrhage. Given the persistent coagulopathy and gastrointestinal hemorrhage, the patient received Factor VIII Inhibitory Bypassing Activity (FEIBA, Baxter) at a dose of 35 $\mathrm{IU} / \mathrm{kg}$. A femoral dialysis catheter was placed under ultrasound guidance. A single-wall puncture technique was used to 
minimize the risk of hemorrhagic and mechanical complications. The patient underwent sustained low-efficiency dialysis in an attempt to eliminate dabigatran in the context of his acute kidney injury. With dialysis, the INR decreased further (see Figure 1), and the gastrointestinal hemorrhage abated, as evidenced by stabilization of hemoglobin concentration and a reduction in transfusion requirements. The patient underwent a second dialysis session on the third day of admission to the ICU.

The patient's clinical course was complicated by shock, hypoxemic respiratory failure, and circulatory overload following massive transfusion. The acute kidney injury resolved, and serum creatinine returned to normal. Repeat endoscopy revealed moderate to severe erosive esophagitis. The patient was transferred to the ward and was ultimately discharged from hospital on the 11th day of admission.

\section{DISCUSSION}

This case of severe hemorrhage complicating anticoagulation with dabigatran underlines several key issues related to the pharmacology of dabigatran and the emergent management of hemorrhagic complications in such patients. First, the development of renal insufficiency had a major impact on the clearance of dabigatran. Second, large volumes of plasma and conventional reversal agents were administered to treat the hemorrhage, but with little clinical effect. All acute care providers should be aware of the inefficacy of standard reversal agents and blood component replacement in this setting. Prompt institution of rational and effective resuscitation is necessary in cases of severe dabigatran-related hemorrhage.

\section{Pharmacology of Dabigatran}

Dabigatran is a specific and reversible direct thrombin inhibitor that inhibits the coagulation cascade by preventing thrombin-mediated events, including cleaving of fibrinogen to fibrin monomers; activation of factors V, VIII, XI, and XIII; and platelet aggregation. Following oral administration, the prodrug dabigatran etexilate is absorbed and converted to dabigatran, with maximum plasma concentrations occurring within 2 h. ${ }^{3}$ Plasma levels of dabigatran may be affected by drugs that affect the transport of dabigatran etexilate across the intestinal wall (such as rifampin, carbamazepine, and amiodarone). About $35 \%$ of circulating dabigatran is bound to plasma proteins, and dabigatran is widely distributed through all body tissues except the brain. ${ }^{3}$ Dabigatran is almost entirely eliminated in the urine, with a very small amount conjugated in the liver and secreted in bile.

Dabigatran is eliminated in the urine primarily in unchanged form $(80 \%)$, with the remainder partially conjugated $(20 \%){ }^{3}$ The half-life of dabigatran in patients with normal renal function is 14 to $17 \mathrm{~h}$, but it can be greater than $24 \mathrm{~h}$ in patients with severe renal impairment. ${ }^{4}$ Pharmacokinetic data

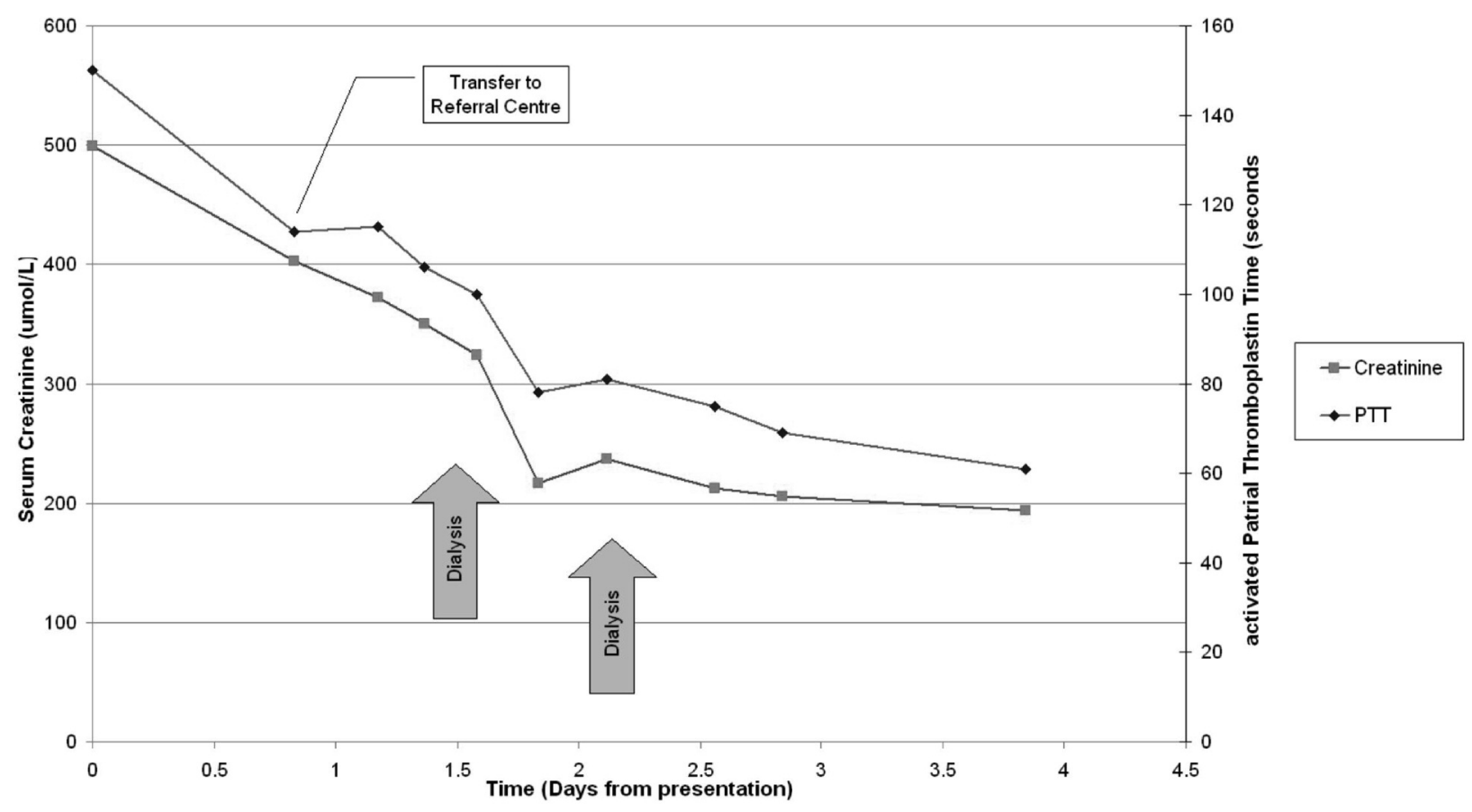

Figure 1. Serum creatinine and partial thromboplastin time over time in a patient with severe dabigatran-related hemorrhage. 
show that, relative to patients with normal creatinine clearance, dabigatran levels are 1.5-fold higher in patients with mild renal impairment (creatinine clearance $67 \mathrm{~mL} / \mathrm{min}$ ), 3.2-fold higher in those with moderate renal impairment (creatinine clearance $41 \mathrm{~mL} / \mathrm{min}$ ), and 6.3-fold higher in those with severe renal impairment (creatinine clearance $24 \mathrm{~mL} / \mathrm{min}$ ). ${ }^{4}$ The dependence on renal elimination is an important consideration in administration of dabigatran to elderly patients, given that age-related decreases in creatinine clearance can result in dabigatran levels almost 2-fold greater in elderly patients than in young healthy patients.

In the case presented here, the patient's gastrointestinal hemorrhage was exacerbated by the preceding development of renal failure (with serum creatinine on presentation of nearly $500 \mu \mathrm{mol} / \mathrm{L}$ ) and resulting accumulation of plasma dabigatran. Although we cannot determine the cause of his renal failure with certainty, it is worth noting that this 80 -year-old man was taking a full dose (150 mg bid) of dabigatran. The 110-mg dose was made available in Canada specifically for patients 80 years of age and older, in light of the age-related decrease in renal function. ${ }^{6}$ The Canadian product monograph suggests that caution be exercised in administering dabigatran to elderly patients and that prescribers should consider this lower dose. There are no specific recommendations on the frequency or extent of monitoring of renal function in these patients, other than that regular assessment of renal status is required.

\section{Management of Severe Hemorrhage in Patients Taking Direct Thrombin Inhibitors}

The management of hemorrhagic complications in patients treated with dabigatran or other direct thrombin inhibitors depends on the severity of the hemorrhage. ${ }^{8}$ Unlike the situation for warfarin and heparin, no specific antidote exists to reverse the effects of dabigatran. A single dose of activated charcoal may be useful in minimizing systemic absorption of dabigatran if administered within $2 \mathrm{~h}$ of ingestion.' General measures in severe hemorrhage include local hemostatic measures such as antifibrinolytics, resuscitation with IV fluids, transfusion of packed red cells, and referral for surgery or interventional radiology as appropriate. Additional management ranges from the discontinuation or delay of the next dose (in cases of minor hemorrhage) to hemodialysis and use of plasma factor concentrates (in cases of severe hemorrhage)..$^{10}$

\section{Monitoring of Hemorrhage Tendency}

Dabigatran prolongs aPTT and thrombin time, whereas the prothrombin time and INR are generally not affected at therapeutic plasma concentrations. ${ }^{8,10}$ The relationship between plasma concentration of dabigatran and aPTT becomes nonlinear at higher doses of dabigatran, and thus neither aPTT nor INR can be used to monitor anticoagulant effect or guide the administration of blood products. ${ }^{8}$ In contrast, the correlation between plasma dabigatran levels and thrombin time is linear. ${ }^{11}$ Therefore, if thrombin time is within normal limits, it is safe to assume that both the plasma level of dabigatran and the risk of hemorrhage are low. ${ }^{12}$

\section{Reversal of Coagulopathy Related to Dabigatran}

There is limited clinical experience with the use of plasma products or other prohemostatic agents in the management of dabigatran-associated hemorrhage. ${ }^{8,13}$ Although frozen plasma is often used in other emergency situations involving significant hemorrhage, there is no evidence or rationale that frozen plasma will reverse the effects of dabigatran (unless there is concurrent dilutional coagulopathy), because the prolongation of prothrombin time and aPTT is due to inhibition, not depletion, of coagulation factors. ${ }^{8,14}$ In making the decision to pursue ongoing transfusion of large volumes of frozen plasma in an attempt to reverse direct thrombin inhibitor-related coagulopathy, the clinician should carefully weigh the limited expected efficacy, the availability of other therapies, and the risks of volume overload and immune-mediated sequelae. Other hemostatic options for management of hemorrhage include administration of recombinant activated factor VII (rFVIIa), prothrombin complex concentrates (PCCs), and FEIBA.

rFVIIa is a potent procoagulant indicated for the treatment of bleeding in hemophilia or congenital factor VII deficiency. Laboratory, animal, and human studies have generated mixed data on its efficacy in reducing hemorrhage associated with dabigatran. ${ }^{15}$ Administration of rFVIIa and hemodialysis were used to manage massive hemorrhage secondary to dabigatran in a patient undergoing cardiac surgery. ${ }^{11}$ Three doses of rFVIIa at $2.4 \mathrm{mg} /$ dose were ineffective, but administration of 2 larger doses $(7.2 \mathrm{mg})$ decreased clinical hemorrhage and allowed postoperative hemodialysis. The risks and benefits of rFVIIa as a hemostatic agent in this context should be considered carefully, given the paucity of data showing efficacy, the risk of thrombotic complications observed with off-label administration of rFVIIa, and the fact that most patients who are receiving dabigatran are at risk for thrombosis. ${ }^{16}$

PCCs contain nonactivated coagulation factors (e.g., Octaplex) and are most often used for the emergent reversal of vitamin $\mathrm{K}$ antagonists (e.g., warfarin). There is one distinct factor complex contratate that contains some activated coagulation factors (FEIBA). FEIBA is indicated for the treatment of hemophilia with inhibitors against factor VIII, and comprises vitamin $\mathrm{K}$-dependent factors II, IX, and X (mainly in nonactivated forms), as well as factor VII (mainly in activated form). 
Both PCCs and FEIBA should theoretically overcome the effect of a direct thrombin inhibitor, but clinical data demonstrating their efficacy in reducing hemorrhage associated with direct thrombin inhibitors are lacking. Dabigatraninduced increases in aPTT and thrombin time were not reversed by PCC in healthy male volunteers who were given dabigatran $150 \mathrm{mg}$ twice daily for 2.5 days followed by a single bolus of PCC (50 IU/kg) or placebo. ${ }^{17}$ Conversely, PCC, but not $\mathrm{FFVII}$, reduced hematoma expansion in an animal model of intracerebral hemorrhage. ${ }^{15}$ FEIBA has shown promise in the reversal of dabigatran in animal models and human studies. ${ }^{18,19}$ FEIBA was more effective than PCC or rFVIIa at correcting direct thrombin inhibitor-induced changes to coagulation parameters in healthy human volunteers, although high doses of $\mathrm{rFVIIa}(120 \mu \mathrm{g} / \mathrm{kg}$, higher than is routinely administered to patients with hemophilia) were also somewhat effective. Although FEIBA may not be stocked at all Canadian hospitals, it is readily available from Canadian Blood Services and can be ordered emergently.

\section{Hemodialysis and Enhanced Elimination}

Dabigatran is dialyzable because of its low molecular weight $(628 \mathrm{~g} / \mathrm{mol})$ and low plasma protein binding (35\%). Multiple sessions of dialysis may be required, however, because of its large volume of distribution. ${ }^{3}$ Data supporting the use of hemodialysis for reversal of dabigatran are derived from small open-label studies performed by the drug's manufacturers. For example, conventional hemodialysis removed $68 \%$ of dabigatran after $4 \mathrm{~h}$ in 6 volunteers with end-stage renal disease who were given a single 50-mg dose of dabigatran. ${ }^{4}$ In another study, reductions of $49 \%-59 \%$ were achieved after conventional hemodialysis, although there was some redistribution of dabigatran following dialysis, leading to a rebound of $8 \%-16 \%{ }^{20}$

Despite the efficacy of hemodialysis in decreasing plasma levels of dabigatran, there are conflicting reports regarding the clinical effectiveness of hemodialysis at achieving hemostasis in patients with major hemorrhage. ${ }^{11,21}$ In one case report, hemodialysis after the use of high-dose rFVIIa resulted in a 3 -fold decrease in dabigatran levels after $6 \mathrm{~h}$ of dialysis, accompanied by cessation of hemorrhage. ${ }^{11}$ In another case, dabigatran-related coagulopathy persisted, despite continuous venovenous hemodialysis. ${ }^{21}$ These case reports underline the need to further clarify the role of dialysis in dabigatranassociated major hemorrhage, particularly the dose, frequency, and mode of dialysis (continuous renal replacement versus intermittent conventional dialysis).

Finally, because dabigatran etexilate is a lipophilic molecule, single-dose oral activated charcoal and charcoal hemoperfusion may be of benefit in reducing plasma levels of dabigatran if administered within $2 \mathrm{~h}$ of ingestion. Large- volume hemoperfusion (i.e., 3.6 plasma volumes) with an activated charcoal cartridge has been shown to reduce dabigatran plasma levels by more than $95 \%$ in vitro. ${ }^{22}$ There are no clinical data supporting the efficacy of hemoperfusion in severe dabigatran-related hemorrhage.

\section{CONCLUSION AND RECOMMENDATIONS}

This report has described a case of severe gastrointestinal hemorrhage secondary to accumulation of dabigatran in a patient who developed acute renal failure. Hemostasis was achieved and coagulopathy ultimately resolved with FEIBA and hemodialysis. The reversal of hemorrhage induced by direct thrombin inhibitors continues to be a vexing problem in which traditional approaches to reversal of oral vitamin $\mathrm{K}$ antagonists (frozen plasma and vitamin $\mathrm{K}$ ) are ineffective. FEIBA appears to be a promising reversal agent, but there are inconsistent data supporting the use of other concentrates, such as rFVIIa and PCC. There appears to be sound rationale for conventional hemodialysis in life-threatening hemorrhage, although clinical data are limited, and the efficacy of other modes of dialysis remains unknown. Activated charcoal may be beneficial in the $2 \mathrm{~h}$ following ingestion. The limited literature suggests that thrombin time can be used to monitor coagulopathy due to dabigatran. Additional studies are urgently required to inform the optimal approach to the management of hemorrhage in these patients. In the meantime, all acute care providers should be prepared with a treatment plan based on the limited evidence currently available. ${ }^{13,14,23}$

\section{References}

1. Ezekowitz MD, Wallentin L, Connolly SJ, Parekh A, Chernick MR, Pogue J, et al.; RE-LY Steering Committee and Investigators. Dabigatran and warfarin in vitamin $\mathrm{K}$ antagonist-naive and -experienced cohorts with atrial fibrillation. Circulation. 2010;122(22):2246-53.

2. Harper P, Young L, Merriman E. Bleeding risk with dabigatran in the frail elderly. N Engl J Med. 2012;366(9):864-6.

3. Stangier J, Clemens A. Pharmacology, pharmacokinetics, and pharmacodynamics of dabigatran etexilate, an oral direct thrombin inhibitor. Clin Appl Thromb Hemost. 2009;15 Suppl 1:9S-16S.

4. Stangier J, Rathgen K, Stähle H, Mazur D. Influence of renal impairment on the pharmacokinetics and pharmacodynamics of oral dabigatran etexilate: an open-label, parallel-group, single-centre study. Clin Pharmacokinet. 2010;49(4):259-68.

5. Stangier J, Stähle H, Rathgen K, Fuhr R. Pharmacokinetics and pharmacodynamics of the direct oral thrombin inhibitor dabigatran in healthy elderly subjects. Clin Pharmacokinet. 2008;47(1):47-59.

6. PRADAXTM (dabigatran etexilate) gains approval in Canada for stroke prevention in atrial fibrillation. Ingelheim (Germany): Boehringer Ingelheim Corporate Headquarters; 2010.

7. PRADAX [product monograph]. Burlington (ON): Boehringer Ingelheim Canada Ltd; 2012.57 p.

8. Van Ryn J, Stangier J, Haertter S, Liesenfeld KH, Wienen W, Feuring M, et al. Dabigatran etexilate-a novel, reversible, oral direct thrombin inhibitor: interpretation of coagulation assays and reversal of anticoagulant activity. Thromb Haemost. 2010;103(6):1116-27.

9. Van Ryn J, Sieger P, Kink-Eiband M, Gansser D, Clemens A. Adsorption of dabigatran etexilate in water or dabigatran in pooled human plasma by activated charcoal in vitro [abstract]. Blood. 2009;114(22):1065. 
10. Hankey GJ, Eikelboom JW. Dabigatran etexilate: a new oral thrombin inhibitor. Circulation. 2011;123(13):1436-50.

11. Warkentin TE, Margetts P, Connolly SJ, Lamy A, Ricci C, Eikelboom JW. Recombinant factor VIIa (rFVIIa) and hemodialysis to manage massive dabigatran-associated postcardiac surgery bleeding. Blood. 2012;119(9): 2172-4.

12. Schulman S, Crowther MA. How I treat with anticoagulants in 2012: new and old anticoagulants, and when and how to switch. Blood. 2012; 119(13):3016-23.

13. Kaatz S, Kouides PA, Garcia DA, Spyropolous AC, Crowther M, Douketis JD, et al. Guidance on the emergent reversal of oral thrombin and factor Xa inhibitors. Am J Hematol. 2012;87 Suppl 1:S141-5.

14. Crowther MA, Warkentin TE. Managing bleeding in anticoagulated patients with a focus on novel therapeutic agents. J Thromb Haemost. 2009;7 Suppl 1:107-10.

15. Zhou W, Schwarting S, Illanes S, Liesz A, Middelhoff M, Zorn M, et al. Hemostatic therapy in experimental intracerebral hemorrhage associated with the direct thrombin inhibitor dabigatran. Stroke. 2011;42(12):3594-9.

16. Levi M, Levy JH, Andersen HF, Truloff D. Safety of recombinant activated factor VII in randomized clinical trials. N Engl J Med. 2010; 363(19):1791-800.

17. Eerenberg ES, Kamphuisen PW, Sijpkens MK, Meijers JC, Buller HR, Levi M. Reversal of rivaroxaban and dabigatran by prothrombin complex concentrate: a randomized, placebo-controlled, crossover study in healthy subjects. Circulation. 2011;124(14):1573-9.

18. Van Ryn J, Ruehl D, Priepke H, Wienen W. Reversibility of the anticoagulant effect of high doses of the direct thrombin inhibitor dabigatran, by recombinant factor VIIa or activated prothrombin complex concentrate [abstract 0370]. Haematologica. 2008;93 Suppl 1:148.

19. Marlu R, Hodaj E, Paris A, Albaladejo P, Crackowski JL, Pernod G. Effect of non-specific reversal agents on anticoagulant activity of dabigatran and rivaroxaban: a randomised crossover ex vivo study in healthy volunteers. Thromb Haemost. 2012;108(2):217-24.

20. Haertter S, Tremmel M, Nehmiz G, Liesenfeld K, Moschetti V, Peters H, et al. Investigation of the elimination of dabigatran by haemodialysis in patients with end stage renal disease (ESRD) [abstract PI-68]. Clin Pharmacol Ther. 2012;91 Suppl 1:S32.

21. Cano EL, Miyares MA. Clinical challenges in a patient with dabigatraninduced fatal hemorrhage. Am J Geriatr Pharmacother. 2012;10(2):160-3.
22. van Ryn J, Neubauer M, Flieg R, Krause B, Storr M, Hauel N, et al. Successful removal of dabigatran in flowing blood with an activated charcoal hemoperfusion column in an in vitro test system [abstract]. Pathophysiol Haemost Thromb. 2010;37 Suppl 1:A94.

23. Cushman M, Lim W, Zakai N. 2011 clinical practice guide on anticoagulant dosing and management of anticoagulant-associated bleeding complications in adults. Washington (DC): American Society of Hematology; 2011.

Ian Plener, MD, is with the Department of Medicine, University of Toronto, Toronto, Ontario.

Sharon Sadry, MD, is with the Department of Medicine, University of Toronto, Toronto, Ontario.

Lee Mozessohn, MD, is with the Division of Hematology, University of Toronto, Toronto, Ontario.

Justin Y Lee, BSCPhm, MD, is with the Department of Medicine, Faculty of Health Sciences, McMaster University, Hamilton, Ontario.

Damen Man, BScPhm, PharmD, is with the Abbotsford Regional Hospital, Abbotsford, British Columbia.

Nadine Shehata, MD, MSc, is with the Division of Hematology, University of Toronto, and the Division of Hematology, Mount Sinai Hospital, Toronto, Ontario.

Jeffrey $\mathbf{M}$ Singh, MD, MSc, is with the Interdepartmental Division of Critical Care Medicine and the Department of Medicine, University of Toronto, Toronto, Ontario.

Competing interests: None declared.

Address correspondence to:

Dr Jeffrey M Singh

Toronto Western Hospital

399 Bathurst Street

2McLaughlin - 411K

Toronto ON M5T 2S8

e-mail: jeff.singh@uhn.ca

\section{Advertisers' Index}

\begin{tabular}{lll}
\hline & Ad Page & Prescribing Information \\
\hline Astellas / Xtandi & 2,3 & 86 \\
\hline Baxter / Corporate & 4 & - \\
\hline Pharmaceutical Partners of Canada / Corporate & OBC & - \\
\hline
\end{tabular}

